\title{
Cloud computing tools for training the next generation of cryo-EM structural biologists
}

\author{
Michael A. Cianfrocco ${ }^{a}$ \\ aLife Sciences Institute, University of Michigan, Ann Arbor, Michigan 48109, USA \\ mcianfro@umich.edu
}

Access to adequate computing resources continues to slow the widespread use of cryo-EM across structural biology. To address this limitation and facilitate the spread of cryo-EM, we have developed publicly available 'off-the-shelf' computing environments on both Amazon Web Services and NSF XSEDE computing infrastructure [1, 2]. These environments provides users with pre-installed single particle cryo-EM software packages and the ability to utilize both GPU and CPU clusters for immediate data processing. Given this framework of computing resources, we built a software environment to provide structural biologists easy access and management of cloud computing resources, called 'cryoem-cloud-tools' [3]. This environment provides users with the ability to launch and control cloud computing resources without the need of learning the intricacies of Amazon Web Services.

Since establishing these resources, we have extended cryoem-cloud-tools to deploy and manage a 'classroom' of cloud computing resources with preinstalled software and pre-downloaded tutorial datasets. This system allows for individual participants to have access to their own dedicated computing workstation, giving participants a large degree of computing freedom. We have implemented this system at the 2018 Cryo-EM Data Processing Workshop at the University of Michigan and have brought this to the 2019 ACA Meeting as a key part of the cryo-EM hands-on workshop.

\section{$\underline{\text { References }}$}

1. Cianfrocco MA, Leschziner AE. Elife. 2015 May 8;4. PMCID: PMC4440898

2. Cianfrocco MA et al. Practice \& Experience in Advanced Research Computing. 2017.

3. Cianfrocco MA et al. J Struct Biol. 2018 Sep;203(3):230-235. PMCID: PMC6091888 